\title{
Invasive versus echocardiographic gradients in degenerated surgical aortic valve prostheses: A multicenter study
}

Amer N. Kadri, MD, ${ }^{\mathrm{a}}$ George Hanzel, MD, ${ }^{\mathrm{b}, \mathrm{c}}$ Sammy Elmariah, MD, ${ }^{\mathrm{d}}$ Francis Shannon, MD, ${ }^{\mathrm{c}, \mathrm{e}}$ Karim Al-Azizi, MD, ${ }^{\mathrm{f}}$ Judith Boura, MS, ${ }^{\mathrm{g}}$ Michael Mack, MD,${ }^{\mathrm{h}}$ and Amr E. Abbas, MD ${ }^{\mathrm{b}, \mathrm{c}}$

\section{ABSTRACT}

Objectives: To compare echocardiographic and invasive mean gradients obtained concomitantly in degenerated bioprosthetic surgical aortic valves (SAVRs).

Methods: In a multicenter study, we compared concomitant echocardiographic and invasive mean gradients of SAVR, obtained before valve-in-valve transcatheter aortic valve replacement in all patients, patients with primary stenosis (AS), primary aortic regurgitation (AR), and mixed aortic valve disease (MAVD), and in small versus large valves ( $\leq$ or $>23 \mathrm{~mm}$ ). Dimensionless index $(\mathrm{DI})$ was calculated in all groups.

Results: In total, 74 patients were included and data presented as median (interquartile range). Echocardiography-catheterization mean gradient discordance was observed in all patients (invasive $=22 \mathrm{~mm} \mathrm{Hg}$ [11-34] vs echocardiographic $=32 \mathrm{~mm} \mathrm{Hg}$ [21-42], $P=.013$ ), small valves (invasive $=15 \mathrm{~mm} \mathrm{Hg}[8-34] \mathrm{vs}$ echocardiographic $=28 \mathrm{~mm} \mathrm{Hg}[21-41], \quad P=.013)$, and large valves (invasive $=20 \mathrm{~mm} \mathrm{Hg}[8.5-27.13]$ vs echocardiographic $=32 \mathrm{~mm} \mathrm{Hg}\left[\begin{array}{ll}25.5 & 41.5\end{array}\right]$, $P<.0001$ ), with a bias of $8 \pm 15 \mathrm{~mm} \mathrm{Hg}$ and wide limits of agreement ( -22 to $39 \mathrm{~mm} \mathrm{Hg}$ ) on Bland-Altman plots, indicating these modalities may not be interchangeable. Discordance occurred in $A R$ (invasive $=3 \mathrm{~mm} \mathrm{Hg}[1-6]$ vs echocardiographic $=12 \mathrm{~mm} \mathrm{Hg}$ [7-22], $P=.017$ ) and in MAVD (invasive $=19 \mathrm{~mm}$ $\mathrm{Hg}[12-29]$ vs echocardiographic $=31 \mathrm{~mm} \mathrm{Hg}$ [23-39], $P<.0001$ ) but not in AS (invasive $=35 \mathrm{~mm} \mathrm{Hg}$ [24-45] vs echocardiographic $=41 \mathrm{~mm} \mathrm{Hg}$ [30-50], $P=.45)$. A lower DI (0.21 [0.14-0.25]) occurred in AS compared with MAVD (0.31 [0.19-0.39]) and AR (0.55 [0.51-0.69]), P < .0001.

Conclusions: Discordance between echocardiography and invasive mean gradients exists in degenerated SAVR, regardless of valve size, but depends on mechanism of failure and DI helps stratify these patients. With a discrepancy between echocardiographic mean gradients AND the patient's symptoms OR the valve leaflet structure and/or mobility on imaging, especially before redo-SAVR or valve-in-valve transcatheter aortic valve replacement, invasive gradients may adjudicate the true valvular hemodynamics. (JTCVS Open 2021;7:51-60)

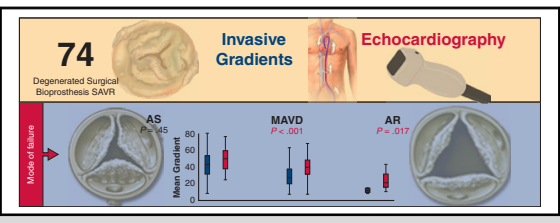

Echocardiography and invasive gradient discordance in degenerated bioprosthetic valves.

\section{CENTRAL MESSAGE}

Echocardiography overestimates invasive aortic gradients in degenerated surgical bioprostheses. Bias and wide limit of agreement on Bland-Altman suggest the modalities cannot be used interchangeably.

\section{PERSPECTIVE}

When there is a discrepancy between the echocardiographic gradients AND the patient's symptoms $O R$ the valve leaflet structure and/or mobility on imaging, especially prior to redosurgical or valve-in-valve transcatheter aortic valve replacement, invasive gradients may adjudicate the true valvular hemodynamics.

\section{See Commentary on page 61.}

\footnotetext{
From the ${ }^{\mathrm{a} D}$ Division of Cardiology, Beaumont Health, Dearborn, Mich; ${ }^{\mathrm{b}}$ Department of Cardiovascular Medicine and ${ }^{\mathrm{e}}$ Division of Cardiovascular Surgery, Beaumont Hospital, Royal Oak, Mich; ${ }^{\mathrm{c} O a k l a n d}$ University William Beaumont School of Medicine, Auburn Hills, Mich; ${ }^{\mathrm{d} D i v i s i o n}$ of Cardiology, Massachusetts General Hospital, Boston, Mass; Divisions of ${ }^{\mathrm{f}}$ Cardiology and ${ }^{\mathrm{h}}$ Cardiac Surgery, The Heart Hospital Baylor Plano, Plano, Tex; and ${ }^{\mathrm{g}}$ Department of General Medical Education, Ascension-Macomb Oakland Hospital, Warren, Mich.

Received for publication Dec 15, 2020; accepted for publication June 4, 2021; available ahead of print Aug 2, 2021

Address for reprints: Amr E. Abbas, MD, Beaumont Hospital, 3601 W 13 Mile Rd, Royal Oak, MI 48073 (E-mail: amr.abbas@beaumont.edu).

2666-2736

Copyright (C) 2021 The Author(s). Published by Elsevier Inc. on behalf of The American Association for Thoracic Surgery. This is an open access article under the CC BY-NC-ND license (http://creativecommons.org/licenses/by-nc-nd/4.0/).

https://doi.org/10.1016/j.xjon.2021.06.029
}

$\checkmark$ Video clip is available online.

While the method of choice for treating failed surgical aortic valve (AV) bioprosthesis via valve-in-valve transcatheter aortic valve replacement (ViV-TAVR) versus redo-surgical aortic valve replacement (SAVR) is based on multiple clinical variables, the evaluation of failed valvular hemodynamics is paramount in both and includes 


$$
\begin{aligned}
\text { Abbreviations and Acronyms } \\
\text { AR }=\text { aortic regurgitation } \\
\text { AS }=\text { aortic stenosis } \\
\text { AV }=\text { aortic valve } \\
\text { AVA }=\text { aortic valve area } \\
\text { DI }=\text { dimensionless index } \\
\text { IQR }=\text { interquartile range } \\
\text { LV }=\text { left ventricle } \\
\text { LVOT }=\text { left ventricular outflow tract } \\
\text { MAVD }=\text { mixed aortic valve disease } \\
\text { SAVR }=\text { surgical aortic valve replacement } \\
\text { TAVR }=\text { transcatheter aortic valve replacement } \\
\text { TVI }=\text { time velocity integral } \\
\text { ViV }=\text { valve-in-valve }
\end{aligned}
$$

the accurate assessment of the transvalvular mean gradient and prosthesis regurgitation. ${ }^{1}$ This assessment has relied mostly on echocardiography, where the transaortic Doppler waveform has been used in the Bernoulli equation to derive the mean prosthesis gradient and in the continuity equation to derive the prosthetic valve area, whereas determining the degree of prosthesis regurgitation relies primarily on an integrated approach to quantify and qualify the degree of regurgitation. Other echocardiographic measures have also been employed, particularly in stenosis determination, as the dimensionless index (DI), which is the ratio of the left ventricular outflow and AV time velocity integrals (TVIs).

Discordance between echocardiography and catheterization in the assessment of transvalvular mean gradient has been reported in native aortic stenosis (AS), following SAVR, TAVR, and ViV-TAVR. ${ }^{2}$ Echocardiography overestimates the invasive mean gradients, and previous studies have suggested that following SAVR, this discordance is related to valve design ${ }^{3-6}$ and degree of prosthesis obstruction, $^{7}$ and it is independent of the aortic dimension and the phenomenon of pressure recovery. ${ }^{2}$ The relationship between echocardiography and catheterization mean gradients has not been investigated in patients with degenerate bioprosthetic valves. We sought to compare echocardiographic and invasive mean gradients in patients with failed surgical bioprosthesis.

\section{METHODS \\ Study Design}

This is a multicenter study of consecutive patients undergoing ViVTAVR at Beaumont Hospital, Royal Oak, Michigan, The Heart Hospital Baylor Plano, Plano, Texas, and Massachusetts General Hospital, Boston, Massachusetts. Each participating center included patients' clinical, echocardiography, invasive, and valve data into a multicenter registry. The inclusion criteria for this analysis were patients with degenerated surgical bioprosthetic valve who were scheduled to undergo ViV-TAVR with available pre-ViV-TAVR echocardiography and invasive mean gradients. Beaumont Hospital was the coordinating center, and investigators had access to all the data entries after submission by participants and responsible for data integrity. Each institution's institutional review board approved the study (2016-209, June 30, 2016; renews annually, date of last renewal June 30, 2020), protected health information was removed from the data, and data-sharing agreements were signed between the coordinating center and participating institutions. The need for informed consent has been waived as there was minimal risk to the privacy of individuals in this retrospective observational study.

\section{Clinical Data}

Patients' clinical data points included age, sex, body mass index, baseline New York Heart Association functional classification, and history of hypertension, dyslipidemia, diabetes, smoking, chronic kidney disease, and coronary artery disease.

\section{Echocardiography}

Commercially available ultrasound machines were used to acquire echocardiographic examinations, and all measurements were performed according to current guidelines. ${ }^{8-10}$ The diameter of the left ventricular outflow tract (LVOT) was measured immediately beneath the left ventricular border of the bioprosthetic valve stent from outer-to-outer border in a parasternal long-axis zoom view. LVOT TVI was obtained by pulse-wave Doppler sample volume, which was positioned just apical to the bioprosthetic valve at approximately the same location as the diameter of the LVOT. Continuous-wave Doppler examinations were performed to obtain the maximum jet velocity, which was traced to calculate the AV velocity and TVI. A modified Bernoulli equation was used to calculate the mean transprosthetic gradient, and the continuity equation was used to calculate the aortic valve area (AVA). The DI was calculated as LVOT TVI/AV TVI. The severity of aortic regurgitation (AR) was derived using a multiparametric approach that included jet width in LVOT with color Doppler, jet deceleration rate with continuous-wave Doppler, presence of diastolic flow reversal in the descending aorta, vena contracta width, jet width/LVOT width percent, and regurgitant volume and fraction.

\section{Invasive Mean Gradient}

Invasive mean gradients were obtained immediately before ViV-TAVR valve deployment. Two transducers were used to assess simultaneous left ventricle (LV) and aortic pressures (pull-back gradients were not used). Both transducers are zeroed, and waveforms were checked for dampening before recording. The aortic pressure was obtained in the ascending aorta via a pigtail catheter introduced through a secondary access or via the aortic lumen of a dual-lumen catheter. The LV pressure was obtained either via the LV lumen of a dual-lumen catheter or via another pigtail introduced into the LV. Echocardiographic and invasive mean gradients were obtained within 15 to 20 minutes of each other.

\section{MAGNITUDE OF DISCORDANCE}

To determine the magnitude of discordance, we calculated the absolute discordance difference (echocardiographic gradient - invasive mean gradient) and the percent discordance difference (echocardiographic gradient invasive gradient)/echocardiographic mean gradient $\times 100$.

\section{Mechanism of Failure}

Patients were stratified according to mechanism of failure including primary bioprosthetic AR, primary bioprosthetic AS, and mixed aortic valve disease (MAVD). Bioprosthetic AR was defined as severe AR (by either echocardiography 
or aortography $\geq 3+$ ), along with a peak aortic velocity $<3 \mathrm{~m} / \mathrm{s}$ or a gradient $<20 \mathrm{~mm} \mathrm{Hg}$ and an AVA $>1.5 \mathrm{~cm}^{2}$. Bioprosthetic AS was defined by the AVA $<1 \mathrm{~cm}^{2}$, demonstration of restricted leaflets by any imaging modality, presence of less than moderate AR, and peak aortic velocity $>3 \mathrm{~m} / \mathrm{s}$, and. Finally, MAVD was defined by the presence of moderate or greater AS and moderate or greater AR. ${ }^{11-13}$ Moderate AS was defined as AVA $1-1.5 \mathrm{~cm}^{2}$ and a gradient of 20 to $40 \mathrm{~mm} \mathrm{Hg}$ or peak velocity 3-4 m/s.

\section{Statistical Analysis}

Descriptive statistics were provided for all data. Missing data remained missing and were not replaced with substitutions or interpolations. Categorical data were presented as count and percentages of occurrence. Continuous data were reported as mean \pm the standard deviation or median with interquartile range (IQR), dependent on the normality of the data.

We examined the difference between the invasive mean gradient and the echocardiographic mean gradient with a sign test of the paired differences. Scatterplots were completed to show the relationship between invasive mean gradient and the echocardiographic mean gradient. Bland-Altman plots were then created to examine possibility of replacing one with the other.

Spearman correlations and scatterplots were used to examine the linear relationships between the paired difference in the invasive versus echocardiographic mean gradient and heart rate. Types of SAVR used, hospital locations, mechanism of failure, and SAVR valve size were examined for paired differences between echocardiographic and invasive mean gradient and DI using either a Wilcoxon rank test for SAVR valve size and a KruskalWallis tests for the others. The paired differences in the invasive versus echocardiographic mean gradient and DI were examined by large versus small SAVR valve size with Wilcoxon rank tests. Statistical analyses were performed with SPSS, version 25.0 (IBM Corp, Armonk, NY) and SAS for Windows (version 9.4, Cary, NC).

\section{RESULTS}

We identified 101 patients with failed bioprosthetic SAVR who underwent ViV-TAVR, with a total of 74 patients who had available concomitant pre-ViV-TAVR echocardiographic and invasive mean gradients obtained within 15 to 20 minutes and known mechanism of failure. Mean age was 74.9 years $( \pm 12)$, the majority of the patients were male $(69 \%)$, and $73 \%$ had New York Heart Association classes of III-IV (Table 1). Further, 41 patients $(55.4 \%$ ) had MAVD, $24(32.4 \%)$ patients had primary severe AS, and only $9(12.2 \%)$ patients had primary AI (Table 2$)$.

In all 74 patients, there was significant absolute discordance between median paired difference (IQR) echocardiographic and invasive mean gradients: $8 \mathrm{~mm} \mathrm{Hg}$ (-1 to 21)
TABLE 1. Baseline characteristics

\begin{tabular}{|c|c|}
\hline $\begin{array}{l}\text { Baseline characteristics } \\
\qquad(\mathrm{N}=\mathbf{7 4}) \\
\end{array}$ & Findings or $\mathbf{N}(\%)$ \\
\hline Age, $\mathrm{n}=74$, mean $( \pm \mathrm{SD})$ & $74.9(12)$ \\
\hline BMI $\left(\mathrm{Kg} / \mathrm{m}^{2}\right), \mathrm{n}=71$, mean $( \pm \mathrm{SD})$ & $29(6)$ \\
\hline $\operatorname{BSA}\left(\mathrm{m}^{2}\right), \mathrm{n}=73$, mean $( \pm \mathrm{SD})$ & $1.96(0.2)$ \\
\hline Male sex, $n=74$ & $51(68.9 \%)$ \\
\hline Coronary artery disease, $\mathrm{n}=74$ & $41(55 \%)$ \\
\hline $\begin{array}{l}\text { History of coronary artery bypass graft } \\
\text { surgery, } n=60\end{array}$ & $13(21.7 \%)$ \\
\hline History of cerebrovascular accidents, $\mathrm{n}=52$ & $12(23 \%)$ \\
\hline Hypertension, $\mathrm{n}=74$ & $55(74 \%)$ \\
\hline Hyperlipidemia, $\mathrm{n}=74$ & $61(82 \%)$ \\
\hline Peripheral arterial disease, $\mathrm{n}=52$ & $5(9.6 \%)$ \\
\hline Diabetes mellitus, $\mathrm{n}=74$ & $28(38 \%)$ \\
\hline Atrial fibrillation, $\mathrm{n}=60$ & $26(43 \%)$ \\
\hline Chronic kidney disease, $\mathrm{n}=74$ & $36(49 \%)$ \\
\hline Chronic obstructive lung disease, $n=60$ & $16(27 \%)$ \\
\hline History of smoking, $\mathrm{n}=74$ & $47(64 \%)$ \\
\hline \multicolumn{2}{|l|}{ Baseline NYHA, $n=73$} \\
\hline I & $1(1.4 \%)$ \\
\hline II & $19(26.0 \%)$ \\
\hline III & $44(60.3 \%)$ \\
\hline IV & $9(12.3 \%)$ \\
\hline
\end{tabular}

$S D$, Standard deviation; $B M I$, body mass index; $B S A$, body surface area; $N Y H A$, New York Heart Association functional classification.

(median invasive $21.53 \mathrm{~mm} \mathrm{Hg}\left[\begin{array}{ll}11 & 34\end{array}\right]$ vs echocardiographic mean-gradient $32 \mathrm{~mm} \mathrm{Hg}$ [21 42], $P<.0001$; Figure 1). A linear relationship between both modalities was observed in Spearman correlation $(0.57, P<.0001$; Figure E1). However, A Bland-Altman plot was created to examine the difference between echocardiographic and invasive mean gradients (bias $=8 \pm 15 \mathrm{~mm} \mathrm{Hg}$ ), with wide limits of agreement ( -22 to $39 \mathrm{~mm} \mathrm{Hg}$ ) and no clear pattern, indicating a poor fit to substitute either modality for one another and thus they may not be considered interchangeable (Figure E2). Furthermore, there is no correlation between heart rate and the degree of discordance $(\mathrm{r}=-0.038, P=.77)$.

\section{Analysis According to SAVR Size}

Among 59 patients with available SAVR size, 31 (53\%) had small and $28(47 \%)$ had large valve size. Absolute discordance was present in large (13 [4-20] $\mathrm{mm} \mathrm{Hg}$, median invasive $20 \mathrm{~mm} \mathrm{Hg}$ [9-27] vs echocardiographic mean gradient $32 \mathrm{~mm} \mathrm{Hg}$ [26 42], $P<.0001)$ and in small $8(-1$ to 23$) \mathrm{mm} \mathrm{Hg}$ (median invasive $15 \mathrm{~mm} \mathrm{Hg}$ [8-34] vs echocardiographic mean gradient $28 \mathrm{~mm} \mathrm{Hg}$ [21-41], $P=.013$ ) (Figure 2, A). Also, there was no difference in discordance 
TABLE 2. Baseline bioprosthetic valve, CT, and echocardiographic characteristics

\begin{tabular}{lc}
\hline \multicolumn{1}{c}{$\begin{array}{c}\text { Variable } \\
(\mathbf{N}=\mathbf{7 4})\end{array}$} & $\begin{array}{c}\text { Findings } \\
\text { median }(\text { IQR) or } \mathbf{N}(\%)\end{array}$ \\
\hline SAVR size, $\mathrm{n}=59$ & $31(52 \%)$ \\
Small $(\leq 23 \mathrm{~mm})$ & $28(48 \%)$ \\
Large $(>23 \mathrm{~mm})$ & \\
SAVR type, $\mathrm{n}=74$ & $15(20.3 \%)$ \\
Trifecta & $14(18.9 \%)$ \\
Carpentier-Edwards/PERIMOUNT & $13(17.6 \%)$ \\
Mosaic & $16(21.6 \%)$ \\
Other & $16(21.6 \%)$ \\
Unknown & $24(32.4 \%)$ \\
Mechanism of failure, $\mathrm{n}=74$ & $9(12.2 \%)$ \\
Aortic stenosis & $41(55.4 \%)$ \\
Aortic regurgitation & $55(45-60)$ \\
Mixed aortic valve disease & $23(32 \%)$ \\
LVEF, $\mathrm{n}=73$ median $(\mathrm{IQR})$ & $34(29-43)$ \\
LVEF $<50 \%$ & $0.90(0.67-1.22)$ \\
\hline SVI, $\mathrm{mL} / \mathrm{m}^{2}, \mathrm{n}=63$, median $(\mathrm{IQR})$ & $0.48(0.32-0.65)$ \\
AVA, $\mathrm{cm}^{2}, \mathrm{n}=61$, median $(\mathrm{IQR})$ & $3.5(3.1-4.3)$ \\
\hline iAVA, $\mathrm{cm}^{2} / \mathrm{m}^{2}$, median $(\mathrm{IQR})$ & \\
$\mathrm{CT}$ annular area, $\mathrm{cm}^{2}, \mathrm{n}=49$, median $(\mathrm{IQR})$ & \\
\hline
\end{tabular}

$C T$, Computed tomography; $I Q R$, interquartile range; $S A V R$, surgical aortic valve replacement; $L V E F$, left ventricular ejection fraction; $S V I$, stroke volume index; $A V A$, aortic valve area; $i A V A$, indexed aortic valve area.

between echocardiographic and invasive mean gradients of large versus small SAVR valve sizes $(P=.70)$.

\section{Analysis According to SAVR Type}

Among the 74 patients included, 15 had Trifecta valve, 14 had Carpentier-Edwards/PERIMOUNT valve, 13 had Mosaic valve, 16 had other type of valves (Biocor,
Freestyle, Hancock, Homograft, Magna Ease, and MitraFlow), and 16 patients had an unknown type of valve. There was no difference in valve sizes between different valve types $(P=.19)$. Also, there was no difference in discordance between echocardiographic and invasive gradients of different valve types $(P=.19)$.

\section{Analysis According to Hospital}

A total of 52 patients underwent ViV-TAVR at Beaumont Hospital, whereas 14 patients and 8 patients underwent the procedures at Massachusetts General Hospital and The Heart Hospital Baylor Plano, respectively. No significant difference was observed when we compared the DI $(P=.18)$ as well as the median paired difference between echocardiographic and invasive mean gradients across all three hospitals $(P=.08$, with a tendency to reach a statistically significant level).

\section{Analysis According to Mechanism of Failure}

Echocardiographic and invasive mean gradient absolute discordance was observed in 41 patients with MAVD (12 [3-22] $\mathrm{mm} \mathrm{Hg}$, median invasive $19 \mathrm{~mm} \mathrm{Hg}$ [12-29] vs echocardiographic mean gradient $31 \mathrm{~mm} \mathrm{Hg}$ [23-39]; $P<.0001$ ) as well as in 9 patients with bioprosthetic AR (7 [5-12] mm $\mathrm{Hg}$, median invasive $3 \mathrm{~mm} \mathrm{Hg} \mathrm{[1-6]} \mathrm{vs} \mathrm{echocardiographic}$ mean gradient $12 \mathrm{~mm} \mathrm{Hg}$ [7-22]; $P=.017)$. However, in 24 patients with bioprosthetic AS (4 [ -10 to 19$] \mathrm{mm} \mathrm{Hg}$, median invasive $35 \mathrm{~mm} \mathrm{Hg}$ [24-45] vs echocardiographic mean-gradient $41 \mathrm{~mm} \mathrm{Hg}$ [30-50]; $P=.45$ ), no discordance was observed (Figure 2, B).

While the absolute discordance difference was not significantly greater in bioprosthetic AR compared with MAVD or bioprosthetic AS, the percent discordance difference was greater in primary AR, 67\% (IQR 44\%-90\%)

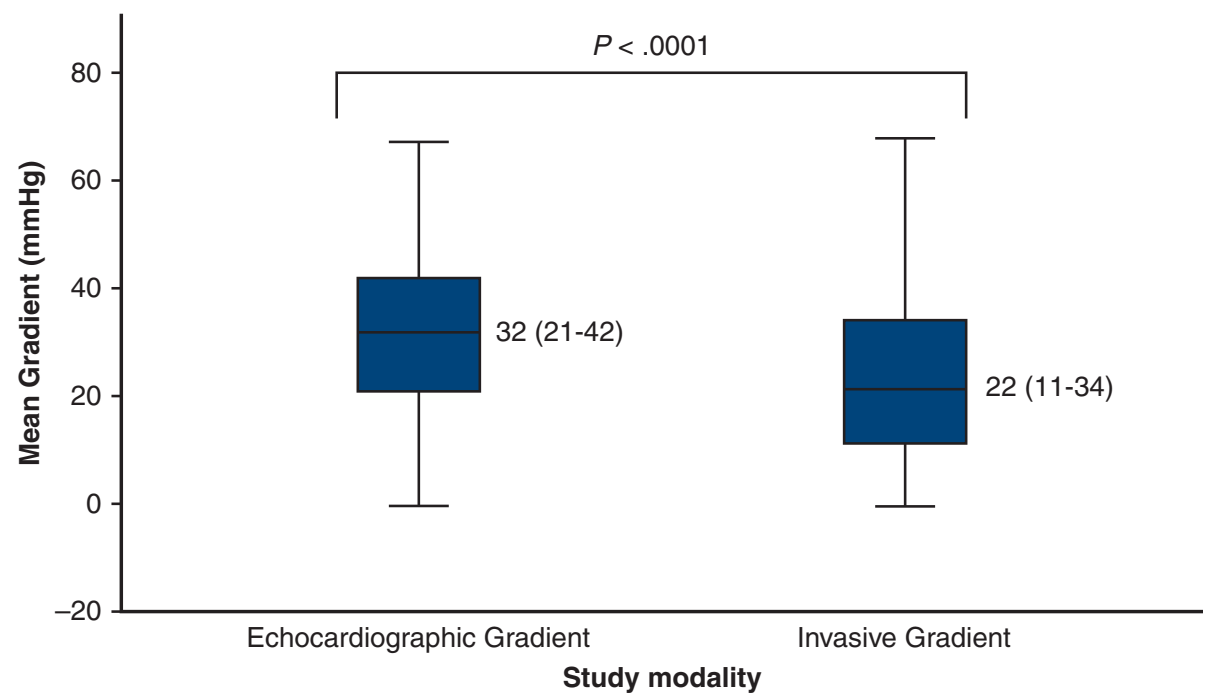

FIGURE 1. Echocardiography significantly overestimated transaortic valve mean gradients compared with invasive mean-gradients in all patients $32 \mathrm{~mm}$ $\mathrm{Hg}$ (interquartile range 21-42) versus $22 \mathrm{~mm} \mathrm{Hg}(11-34)$, respectively, $P<.0001$ (with a sign test of the paired difference with median 8 [ -1 to 21$] \mathrm{mm} \mathrm{Hg}$ ). 


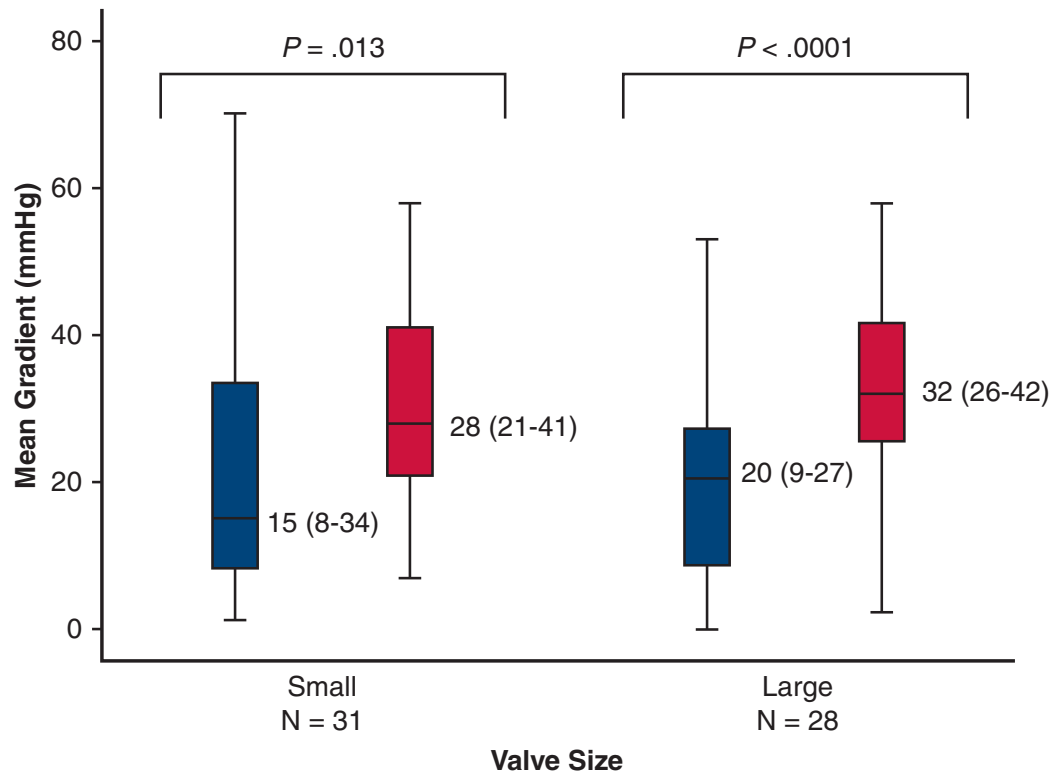

A

Invasive Gradient $\square$ Echocardiographic Gradient

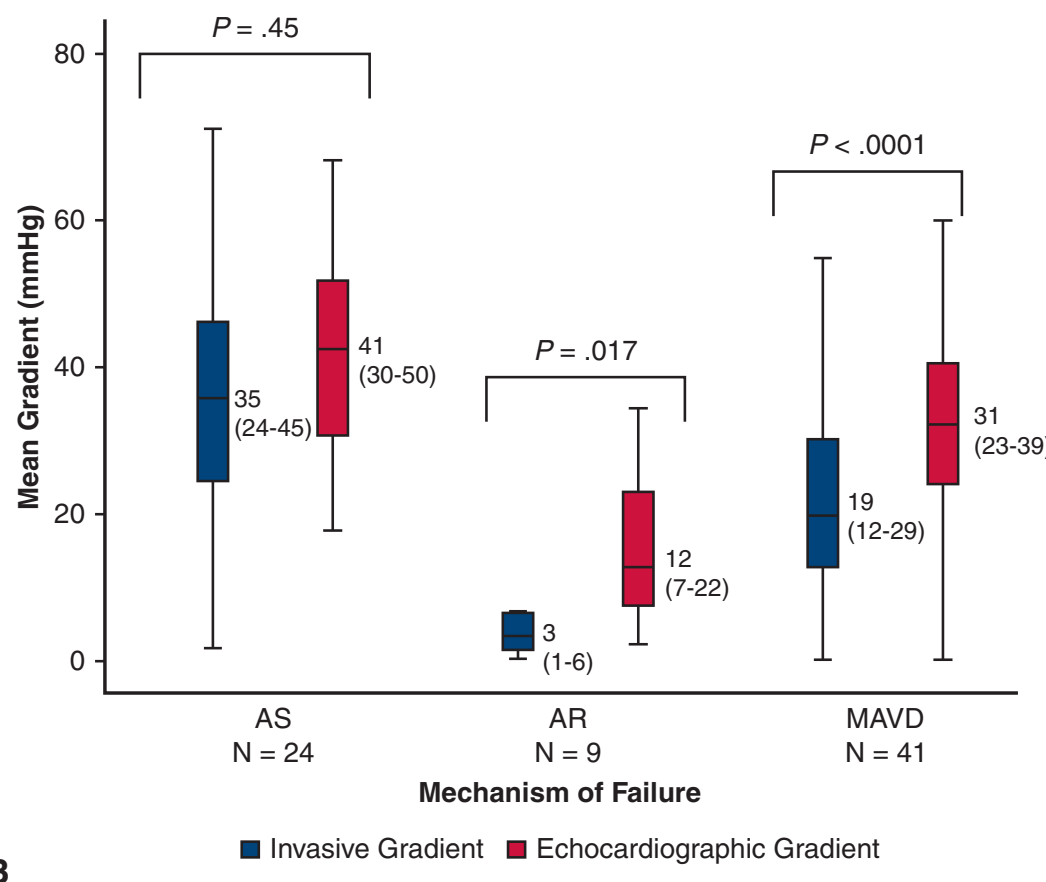

FIGURE 2. A, Echocardiography significantly overestimated transaortic valve mean gradients compared with invasive mean gradients in all patients regardless of valve size in small $(\leq 23 \mathrm{~mm}$ ) (with a sign test of the paired difference with median 8 [ -1 to 23$] \mathrm{mm} \mathrm{Hg}$ ) and large (>23 mm) valves (with a sign test of the paired difference with median 13 [4-20] $\mathrm{mm} \mathrm{Hg}$ ). B, Echocardiography significantly overestimated transaortic valve mean gradients compared with invasive mean gradients in MAVD (with a sign test of the paired difference with median of 12 [3-22] mm Hg), and in primarily AR (with a sign test of the paired difference with median of 7 [5-12] mm Hg), but not in patients with primarily prosthetic AS (with a sign test of the paired difference with median of 4 [ -10 to 19] $\mathrm{mm} \mathrm{Hg}$ ). AS, Aortic stenosis; $A R$, aortic regurgitation; MAVD, mixed aortic valve disease. 


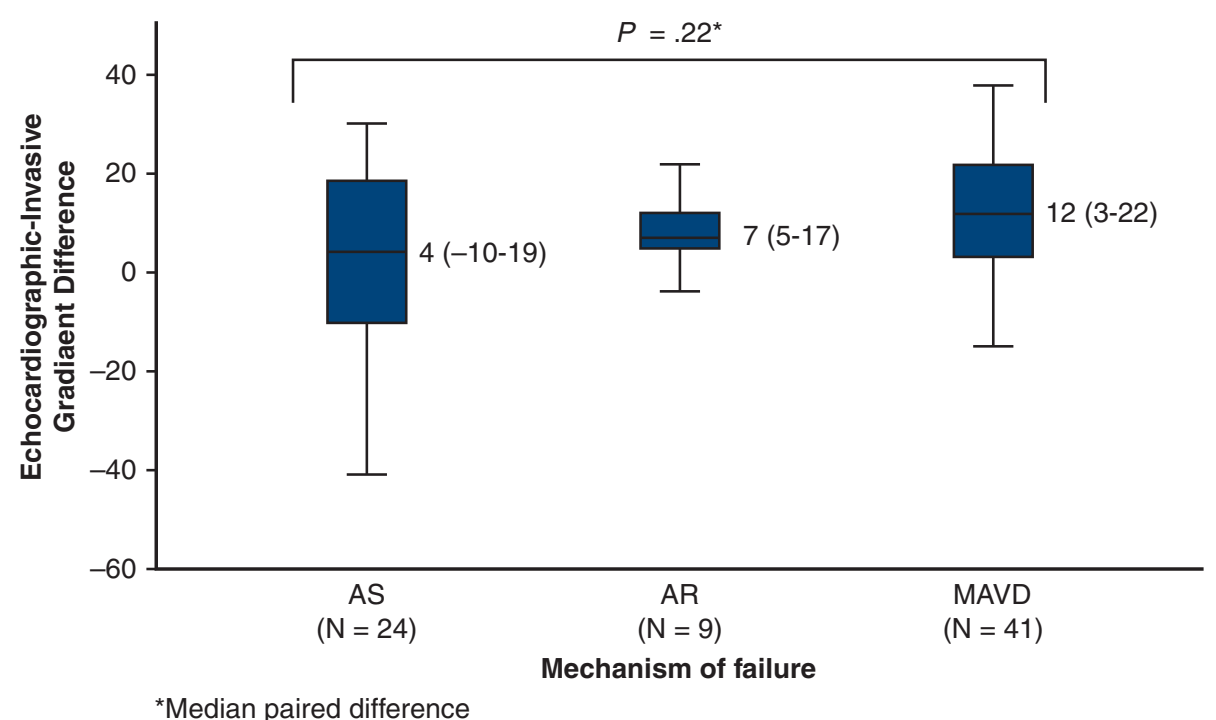

A

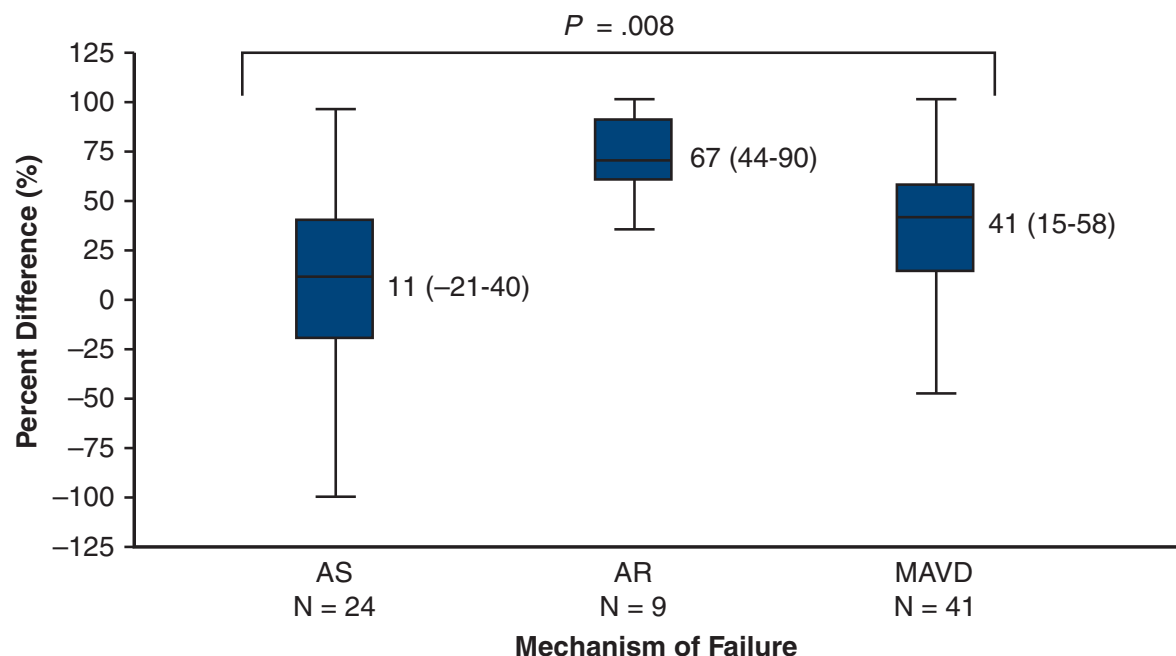

B

FIGURE 3. While the absolute discordance difference (A) was not significantly greater in bioprosthetic AR compared with MAVD or bioprosthetic AS, the percent discordance difference (B) was greater in primary AR compared with MAVD and primary AS. AS, Aortic stenosis; AR, aortic regurgitation; MAVD, mixed aortic valve disease.

compared with MAVD, $41 \%$ (IQR $15 \%-58 \%$ ) and primary AS, $11 \%$ (IQR $-21 \%$ to $40 \%$ ), $P=.008$ (Figure 3 ).

\section{Analysis of the DI}

There was no difference in the DI between large $(0.30$ [0.19-0.40]) vs small (0.28 [0.20-0.40]) SAVR $(P=.89)$. However, patients with bioprosthetic AS exhibited a lower DI (0.21 [0.14-0.25]) compared with MAVD (0.31 [0.190.39]) and bioprosthetic AR (0.55 [0.51-0.69]) $(P<.0001$; Table 3$)$. The DI was not different between hospitals $(P=.18)$. Furthermore, a DI of $\leq 0.25$ was present in $75 \%$ of bioprosthetic AS, $41 \%$ MAVD, and in no patients with bioprosthetic AR $(P=.003)$.

\section{DISCUSSION}

Our study demonstrates that (1) echocardiography overestimates AV mean gradients in degenerated bioprosthetic SAVR valves and a significant discordance occurs between invasive and echocardiographic meangradients regardless of valve size. (2) Discordance depends on the mechanism of SAVR valve failure and the percent discordance progressively increases with diminishing degree of prosthesis stenosis, as with MAVD and AR (Figure 4). (3) A DI $\leq 0.25$ on echocardiogram identifies the majority of patients with primary bioprosthetic AS and excludes all patients with primary bioprosthetic AR (Video 1). 
TABLE 3. Dimensionless valve index in all patients and according to valve size and prosthetic valve failure mechanism

\begin{tabular}{|c|c|c|c|c|}
\hline Paired differences & $\begin{array}{l}\text { All patients } \\
\qquad \mathbf{N}=\mathbf{5 2}\end{array}$ & $\begin{array}{c}\text { Large SAVR } \\
\mathbf{N}=\mathbf{2 6}\end{array}$ & $\begin{array}{c}\text { Small SAVR } \\
\mathbf{N}=\mathbf{2 5}\end{array}$ & $P$ value \\
\hline DI & $0.28(0.19-0.40)$ & $0.30(0.19-0.40)$ & $0.28(0.20-0.40)$ & $\mathrm{N}=51$ \\
\hline \multirow[t]{2}{*}{ Median (IQR) } & & & & $P=.89$ \\
\hline & $\begin{array}{c}\text { AS } \\
\mathbf{N}=\mathbf{1 2}\end{array}$ & $\begin{array}{c}\mathbf{A R} \\
\mathbf{N}=\mathbf{8}\end{array}$ & $\begin{array}{l}\text { MAVD } \\
\mathbf{N}=\mathbf{3 2}\end{array}$ & \\
\hline DI & $0.21(0.14-0.25)$ & $0.55(0.51-0.69)$ & $0.31(0.19-0.39)$ & $\mathrm{N}=52$ \\
\hline Median (IQR) & & & & $P<.0001$ \\
\hline
\end{tabular}

$S A V R$, Surgical aortic valve replacement; $D I$, dimensionless index; $I Q R$, interquartile range; $A S$, aortic stenosis; $A R$, aortic regurgitation; $M A V D$, mixed aortic valve disease.

\section{Discordance Between Echocardiography and Invasive Mean Gradients}

While the invasive gradient is directly measured, the AV velocity Doppler trace is used to derive the prosthetic valve gradient, in echocardiography, by using the simplified Bernoulli equation and the AVA using the continuity equation. As such, an overestimation of the AV velocity will lead to an overestimation of the gradient and underestimation of the

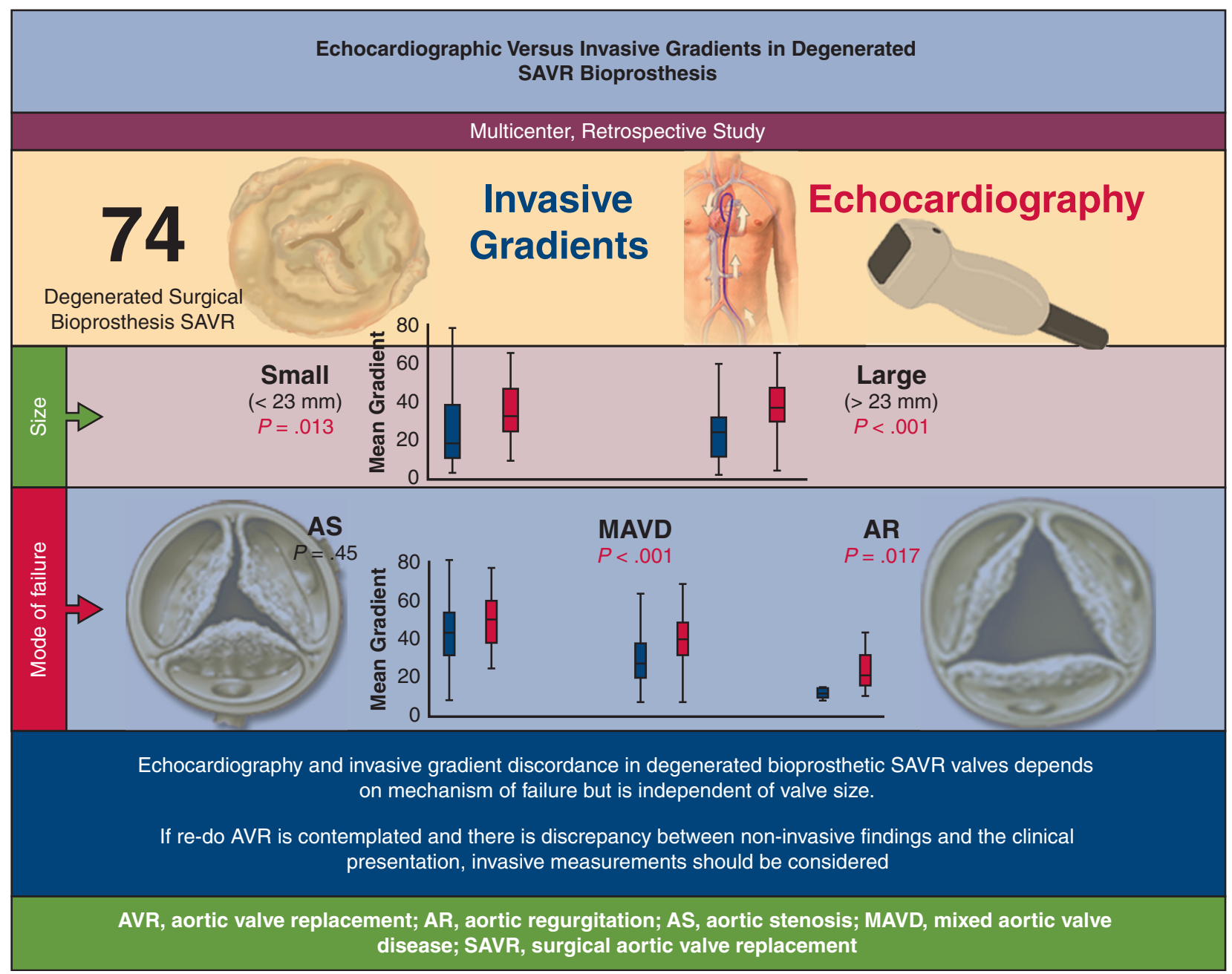

FIGURE 4. Discordance between echocardiography-derived and directly measured invasive mean gradients exists in degenerated bioprosthetic surgical valves, regardless of valve size, and the percent discordance depends on the mechanism of failure. Invasive hemodynamics should be obtained before redoAVR, when it is contemplated by either redo SAVR or ViV-TAVR and when there is discrepancy between elevated echocardiographic mean-gradients and the clinical presentation, when the dimensionless index $>0.25$, and when the valve leaflet structure and mobility do not suggest degenerative bioprosthetic morphology. Invasive assessment should not be used for routine bioprosthetic surveillance, especially in the absence of abnormal echocardiographic hemodynamics. 


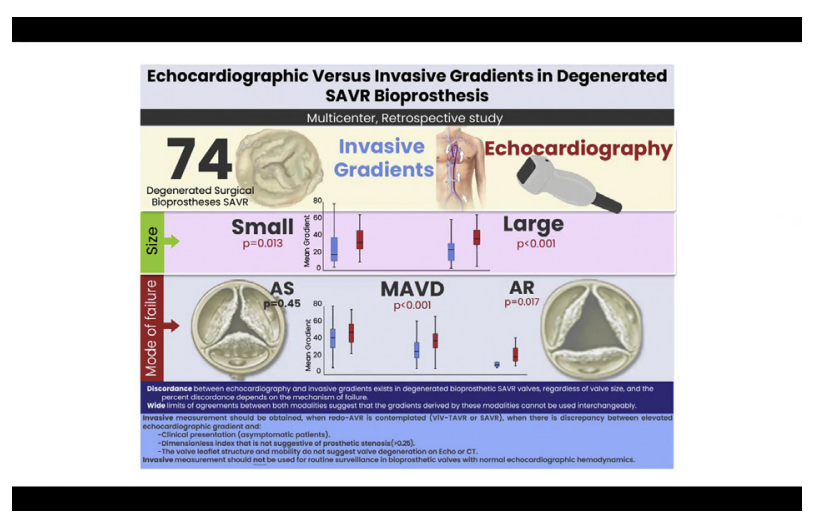

VIDEO 1. In patients with failed bioprosthetic surgical aortic valve, discordance between echocardiographic and invasive mean gradients was observed, regardless of the valve size, when the mechanism of failure is primary aortic regurgitation and mixed aortic valve disease, and to less extent in primary aortic stenosis. Video available at: https://www.jtcvs.org/article/ S2666-2736(21)00189-3/fulltext.

AVA. ${ }^{14}$ Discordance between echocardiographic and invasive gradient has been reported in native severe AS and in prosthetic valves. ${ }^{2}$ Following SAVR, echocardiography overestimates the invasive mean gradient and this is related to the degree of prosthesis stenosis, ${ }^{7}$ valve design and flow pattern, ${ }^{3-6}$ and is independent of ascending aortic area and hence not just a phenomenon of pressure recovery. ${ }^{2}$

This has been also described following TAVR and ViVTAVR $^{15}$ and occurs due to several reasons. First, the Bernoulli equation does not account for the pressure recovery phenomenon. Second, the Bernoulli equation has been modified to omit the nonconvective forces of flow acceleration and viscous forces, attributing their contribution to increase in velocity to convective acceleration, and simplified to omit the LVOT gradient. ${ }^{2}$ While these factors also occur in the presence of valve stenosis, native or prosthetic, their relative contribution is less as most of the velocity is channeled through convective forces and increased velocity is related to a decrease in valve area. ${ }^{14,15}$ However, in the absence of significant valve stenosis, the modifications and simplification of the Bernoulli equation play a larger role, as the increased velocity is not primarily related to an increase in valve mean gradient secondary to a decrease in valve area. Finally, other factors that increase transaortic velocity, such as significant AR, may also be reflected as an increase in mean gradient in the absence of true valve stenosis. Our study suggests that the DI can identify and adjudicate most patients with primary bioprosthetic stenosis and exclude all patients with no bioprosthetic stenosis and in primary bioprosthetic AR. Simply stated, echocardiography overestimates the prosthetic AV mean gradient compared with catheterization in degenerated surgical bioprosthesis except in the presence of pure AS. Moreover, despite a good correlation between both modalities, they may not be used interchangeably.

\section{Earlier In Vitro Studies on Discordance Between Echocardiographic and Invasive Mean Gradients Following SAVR}

Earlier in vitro studies suggested that gradient discordance was minimal in bioprosthetic Hancock valves, whereas it was significant in St Jude mechanical valves. ${ }^{4}$ Moreover, the discrepancy was greater in smaller-sized valves and independent of the design. ${ }^{3,4}$ In an in vitro mechanical valve model of gradually increasing degree of stenosis, the discordance between invasive and echocardiographic mean gradients was found to gradually diminish as the degree of stenosis progressed. ${ }^{7}$ Our study extends this phenomenon to degenerated bioprosthetic SAVR valves and demonstrates that the discordance between invasive and echocardiographic mean gradients progressively increases with diminishing degree of prosthesis stenosis, as with MAVD and AR and that discordance exists regardless of SAVR size.

\section{Clinical Implications}

Our study confirms significant discordance between echocardiography-derived and direct-invasive mean gradient measurements across degenerated bioprosthetic valves and has significant implications before considering redo SAVR or ViV-TAVR in patients with elevated echocardiographic mean gradients across these valves, especially in the presence of MAVD. This discordance occurs regardless of valve size, progressively increases with diminishing degree of stenosis as occurs in the presence of MAVD and primary AR and points to the limitations of echocardiography in accurately determining prosthetic mean gradients in the absence of significant prosthesis stenosis. Accordingly, when there is a discrepancy between the echocardiographic mean gradients AND the patient's clinical status or anatomical structure of the prosthetic valve and leaflet mobility on echocardiography or cardiac computed tomography, especially when redo-SAVR or ViV-TAVR is recommended, invasive mean gradients would help adjudicate the true valvular hemodynamics. In addition, before ViV-TAVR in the operative suite, invasive mean gradients maybe considered before the procedure, especially when the DI do not support valvular stenosis and there is no significant AR. However, routine surveillance with invasive gradients on normal prosthetic valves with normal echocardiographic hemodynamics is not warranted, given the inherent logistic difficulties and procedural risks potentially associated with the invasive procedure.

\section{Limitations}

A core laboratory did not analyze the echocardiographic or invasive data that were determined at the level of the institution. However, careful attention to the fidelity of both echocardiographic and invasive measurements was 
conducted and confirmed with multiple windows for echocardiography and careful balancing and flushing of the transducer for invasive measures.

Moreover, Doppler echocardiography measurements are influenced not only by the anatomy (AS, MAVD, and AR) but also by the physiological state (heart rate, myocardial function, or cardiac output). However, invasive and echocardiographic measurements were obtained under similar hemodynamic conditions, which minimize the effect of these confounders. Furthermore, surgical implantation technique (supra- or intra-annular) can also potentially affect Doppler parameters. It should be also noted that the margins of tissue annulus ranges can vary for different prosthetic valve models having the same labeled valve size. This lack of standardization renders the direct comparison of different valves based on labeled valve size alone not possible.

Finally, patients were supine for echocardiographic examination and underestimation of the mean gradient may still be a possibility, given the patient's position. Thus, the extent of echocardiography and invasive mean gradient discordance may actually be higher.

\section{CONCLUSIONS}

Discordance between echocardiography and invasive mean gradients exists in degenerated bioprosthetic SAVR valves, regardless of valve size, and the mean gradients derived by these modalities cannot be used interchangeably. The percent discordance depends on the mechanism of failure. However, a DI $\leq 0.25$ helps further stratify these patients in the majority of cases with primary bioprosthetic AS and excludes all patients with no significant prosthesis stenosis and in primary bioprosthetic AR. When there is a discrepancy between the echocardiographic mean gradients AND the patient's symptoms OR the bioprosthetic valve leaflet structure and/or mobility on cardiac imaging, especially before redo-SAVR or ViV-TAVR, invasive mean gradients maybe necessary to adjudicate the true valvular hemodynamics.

\section{Conflict of Interest Statement}

A.A. is a consultant for Edwards Lifesciences (modest) and has received research grants (significant). G.H., K.A., and M.M. are consultants for Edwards Life Sciences. S.A. has received research funds from Medtronic and Abbott. All other authors reported no conflicts of interest.

The Journal policy requires editors and reviewers to disclose conflicts of interest and to decline handling or reviewing manuscripts for which they may have a conflict of interest. The editors and reviewers of this article have no conflicts of interest.
We acknowledge Ahmed Ali, MD, Sara Khodor, MD, and Alessandro Vivacqua, MD, for their contributions. Data collection was conducted by individuals with no conflicts of interest, and the statistical analysis was performed by an independent statistician (Judith Boura, MS), who had no conflict of interest. In addition, the conflicts of interest of the individuals with conflict does not include surgical valves and has no impact on the findings of the study.

\section{References}

1. Abbas AE, Pibarot P. Physiological basis for area and gradient assessment: hemodynamic principles of aortic stenosis. In: Abbas AE, ed. Aortic Stenosis: CaseBased Diagnosis and Therapy. London: Springer-Verlag; 2015:29-48.

2. Abbas AE, Mando R, Hanzel G, Goldstein J, Shannon F, Pibarot P. Hemodynamic principles of prosthetic aortic valve evaluation in the transcatheter aortic valve replacement era. Echocardiography. 2020;37:738-57.

3. Baumgartner H, Schima H, Kühn P. Discrepancies between Doppler and catheter gradients across bileaflet aortic valve prostheses. Am J Cardiol. 1993;71:1241-3.

4. Baumgartner H, Khan S, DeRrobertis M, Czer L, Maurer G. Discrepancies between Doppler and catheter gradients in aortic prosthetic valves in vitro: a manifestation of localized gradients and pressure recovery. Circulation. 1990;82: $1467-75$.

5. Baumgartner H, Khan SS, DeRobertis M, Czer LS, Maurer G. Doppler assessment of prosthetic valve orifice area: an in vitro study. Circulation. 1992;85: 2275-83.

6. Baumgartner H, Khan S, DeRobertis M, Czer L, Maurer G. Effect of prosthetic aortic valve design on the Doppler-catheter gradient correlation: an in vitro study of normal St. Jude, Medtronic-Hall, Starr-Edwards and Hancock valves. J Am Coll Cardiol. 1992;19:324-32.

7. Baumgartner H, Schima H, Kuhn P. Effect of prosthetic valve malfunction on the Doppler-catheter gradient relation for bileaflet aortic valve prostheses. Circulation. 1993;87:1320-7.

8. Lang RM, Badano LP, Mor-Avi V, Afilalo J, Armstrong A, Ernande L, et al. Recommendations for cardiac chamber quantification by echocardiography in adults: an update from the American Society of Echocardiography and the European Association of Cardiovascular Imaging. Eur Heart J Cardiovasc Imaging. 2015;16: 233-71.

9. Mitchell C, Rahko PS, Blauwet LA, Canaday B, Finstuen JA, Foster MC, et al. Guidelines for performing a comprehensive transthoracic echocardiographic examination in adults: recommendations from the American Society of Echocardiography. J Am Soc Echocardiogr. 2019;32:1-64.

10. Baumgartner H, Hung J, Bermejo J, Chambers JB, Edvardsen T, Goldstein S, et al. Recommendations on the echocardiographic assessment of aortic valve stenosis: a focused update from the European Association of Cardiovascular Imaging and the American Society of Echocardiography. J Am Soc Echocardiogr. 2017;30:372-92.

11. Barbanti M, Costa G, Zappulla P, Todaro D, Picci A, Rapisarda G, et al. Incidence of long-term structural valve dysfunction and bioprosthetic valve failure after transcatheter aortic valve replacement. J Am Heart Assoc. 2018;7:1-10.

12. Egbe AC, Poterucha JT, Warnes CA. Mixed aortic valve disease: midterm outcome and predictors of adverse events. Eur Heart J. 2016;37:2671-8.

13. Isaza N, Desai MY, Kapadia SR, Krishnaswamy A, Rodriguez LL, Grimm RA, et al. Long-term outcomes in patients with mixed aortic valve disease and preserved left ventricular ejection fraction. J Am Heart Assoc. 2020;9:e014591.

14. Zoghbi WA, Chambers JB, Dumesnil JG, Foster E, Gottdiener JS, Grayburn PA, et al. Recommendations for evaluation of prosthetic valves with echocardiography and Doppler ultrasound. A report from the American Society of Echocardiography's guidelines and standards committee and the task force on prosthetic valves, developed in conjunction. J Am Soc Echocardiogr. 2009;22: 975-1014.

15. Abbas AE, Hanzel G, Shannon F, Gallagher M, Mando R, Chaddha A, et al. PostTAVR trans-aortic valve gradients: echocardiographic versus invasive measurements. Struct Hear. 2019;3:348-50.

Key Words: degenerated bioprosthetic valves, SAVR, echocardiography, hemodynamics, discordance 


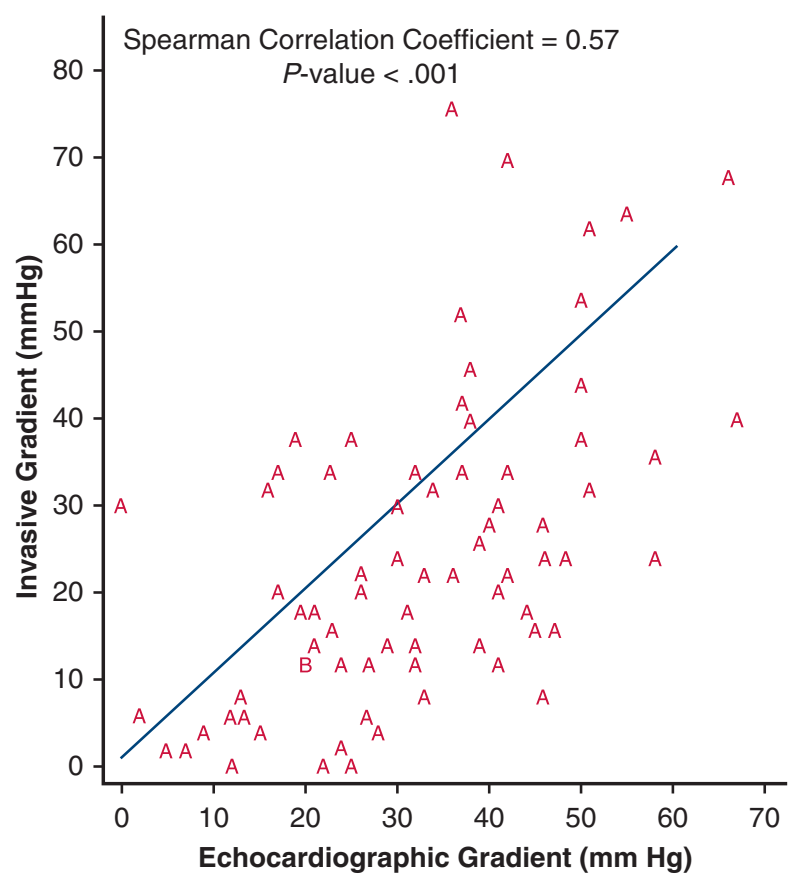

FIGURE E1. There was good correlation between echocardiographic and invasive gradients with a correlation coefficient $\mathrm{r}=0.575$ and $P<.0001$.

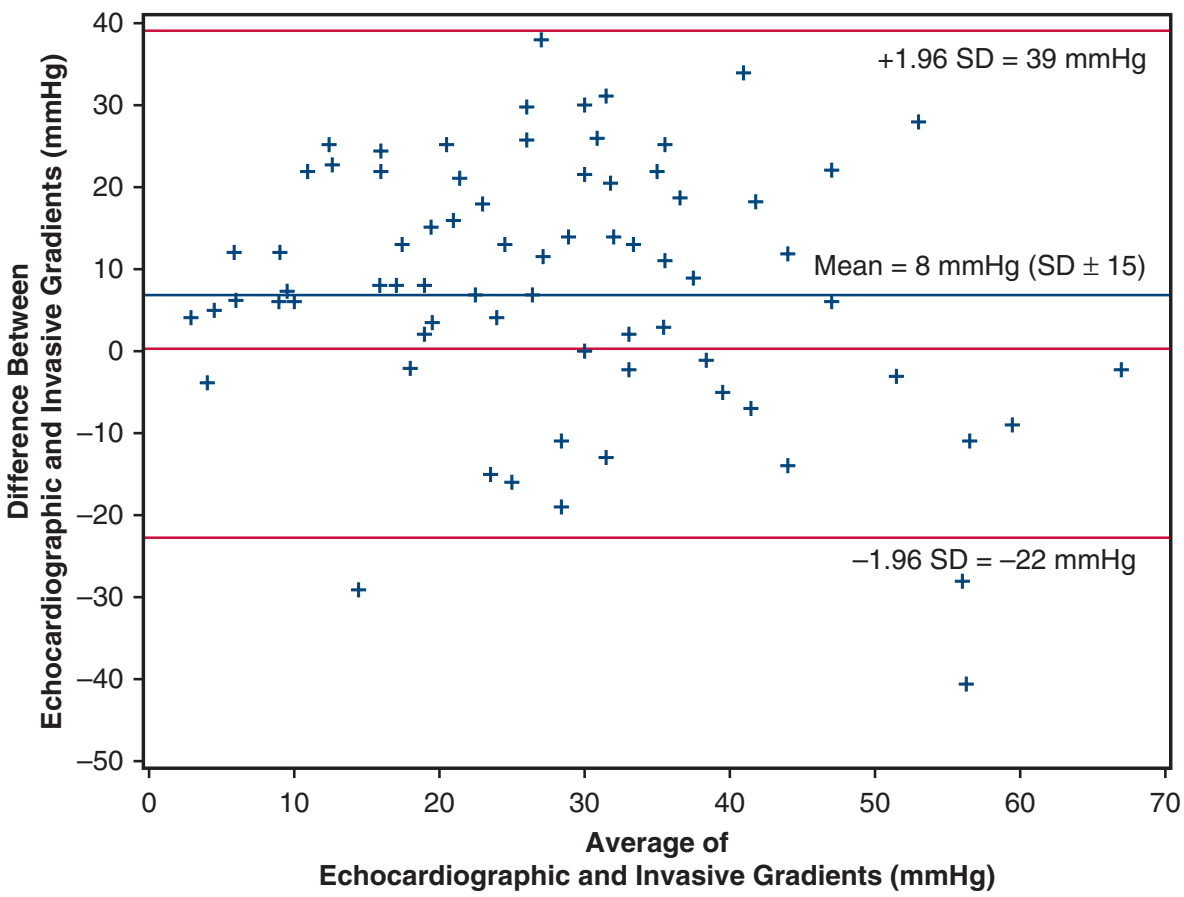

FIGURE E2. A Bland-Altman plot suggests a bias $=8 \pm 15 \mathrm{~mm} \mathrm{Hg}$, with wide limits of agreement ( -22 to $39 \mathrm{~mm} \mathrm{Hg}$ ) and no clear pattern. indicating a poor fit to substitute either modality for one another and thus cannot be considered interchangeable. $S D$, Standard deviation. 